\title{
Lifetime of a soluble solid particle in a stagnant medium: approximate analytical modelling involving fractional (half-time) derivatives
}

\author{
Jordan Hristov \\ University of Chemical Technology and Metallurgy, Department of Chemical Engineering, Sofia 1756, 8 Kliment Ohridsky, \\ blvd. Bulgaria, e-mail: jordan.hristov@mail.bg, http://hristov.com/jordan
}

\begin{abstract}
Approximate analytical solutions concerning lifetime of soluble solid particles in an unbounded stagnant medium have been developed by simple application of fractional half-time derivative in the Riemann-Liouville sense to express the relationship between the net surface mass flux and the concentration at the interface. The solutions start with the initial formulation of Rice and Do on the time-depletion of the radius of a spherical particle expressed through terms including the solubility parameter as the only key parameter controlling the process of dissolution. The two approximate developed solutions use different scaling and dimensionless variables: The $1^{\text {st }}$ solution is developed by an introduction of a similarity variable $\eta=R / \sqrt{D t}$ while the $2^{\text {nd }}$ solution applies the classical scaling using the initial sphere radius as a length scale that leads to dimensionless radius $r=R / R_{0}$ and time $\tau=D t / R_{0}{ }^{2}$. Both solutions provide approximate relationships close to that of Rice and Do.
\end{abstract}

Keywords: particle dissolution, analytical model, fractional half-time derivative.

\section{INTRODUCTION}

A Particle dissolution is an old problem ${ }^{1}$ related to drug dissolution ${ }^{2,3}$, mineral leaching ${ }^{4}$, chemical processing $^{5,6,7}$, controlled drug release $8,9,10$, etc. It is still under intense investigation $3,11,12,13,14,15$. The solution to diffusion-dependent and moving boundary problems ${ }^{16}$ is not an easy task and both numerical ${ }^{17}$ and analytical approaches have been employed ${ }^{1 \mathbf{1}}$. The analytical modeling of solid dissolution ${ }^{11,18}$ is an attractive area because the final relationships of the solutions are in closed forms allowing easily estimating relationships between the key parameters of the process. The modeling approaches employ perturbation solutions ${ }^{19,20}$, the integral balance method $^{21}$, heat transfer analogy ${ }^{22}$ etc.

The present report refers to the calculation of the lifetime of solid particles in stagnant unbounded media, a problem forever young since the time of Noyes and Whitney. The lifetime ${ }^{23}$ is important to many clinical investigations of drugs and industrial processes of dissolving species or minerals. In most cases the concentration field in the surrounding fluid is not of primary interest but only the time of complete dissolution should be estimated. Rice and $\mathrm{Do}^{24}$ developed an elegant analytical solution for a single sphere expressed through the term of solids solubility factor. This solution was the starting point of the present approximate solutions using initial formulation of Rice and $\mathrm{Do}^{24}$ and expressing the particle lifetime in terms involving the solubility factor as a process parameter.

\section{PROBLEM STATEMENT}

\section{Governing equations}

Consider a spherical particle of radius $R$ changing its radius due to dissolution in a stagnant liquid by simple diffusion alone

$\rho\left(\pi \frac{4}{3}\right) \frac{\mathrm{d}\left(R^{3}\right)}{\mathrm{d} t}=-4 \pi R^{2}(t) N_{r=R} R(t=0)=R_{0}$

The net flux of mass to the surrounding medium is
$N_{r=R}=-\left.\frac{D}{1-C^{*} / \rho_{0}} \frac{\partial C}{\partial r}\right|_{r=R}$

while the concentration filed in the stagnant liquid is described by

$D \frac{1}{r^{2}} \frac{\partial}{\partial}\left(r^{2} \frac{\partial C}{\partial r}\right)=\frac{\partial C}{\partial t}, \quad R \leq r \leq \infty$

$C=C^{*}, r=R$

$C \rightarrow C_{0}, r \rightarrow \infty$

$C=C_{0}, t=0, r>R$

This problem has been solved by Rice and $\mathrm{Do}^{24}$ by developing the solution of the model (2) and then expressing $\partial C / \partial r$ at $r=R$ in order to define $N_{r=R}$. The present work refers to the potential of the fractional calculus $^{25,26,27}$ to avoid the entire domain solutions in diffusion problems because the relationship s between the function value and its derivative can be established at the interface of the body and the surrounding medium by the Riemann-Liouville fractional derivative. The approach has seen successfully applied for estimating heat resistances of contacting bodies ${ }^{24}$ by means of semi-derivatives and semi-integrals.

Hence, the present note refers to a new approach in analytical solution of problems relevant to dissolution of solids in unbounded and understated medium. With this approach, the determination of the concentration field outside the particle boundary is unnecessary and the surface concentration gradient should only be known.

\section{Solution strategy}

The classical solution of the problem addresses two consequent steps:

1. Determination of the surface mass flux (1b) through the solution of the problem $(2 \mathrm{a}-2 \mathrm{~d})$, which is a classical semi-infinite diffusion problem ${ }^{11,29}$.

2. Determination of the radius of the sphere undergoing dissolution as a function of the time through (1a). The first step needs determination of the gradient $(\partial C / \partial r)_{r=R}$ in the solid-liquid interface through the solution of the problem $(2 \mathrm{a}-2 \mathrm{~d})$ for the liquid phase. Then, the ordi- 
nary differential equation (1a) concerning $R(t)$ has to be solved as a second step.

The present approach avoids development of the concentration distribution in the bulk phase (step 2) as it was done by Rice and $\mathrm{Do}^{24}$. It directly links the surface concentration and the gradient $(\partial C / \partial r)_{r=R}$ through the Riemann-Liouville fractional derivative. Moreover, the solution concerning the sphere radius (1a) defining the time of complete dissolution is solved in terms of a similarity variable $\eta=R / \sqrt{D t}$ because the semi-infinite diffusion problem in the liquid phase $(2 \mathrm{a}-\mathrm{d})$ has no definite length scale ${ }^{11,29}$.

\section{PROBLEM SOLUTION}

\section{Surface flux: a solution by fractional calculus}

Classically, by the transforms, $\theta=C-C^{*}$ and $u=\theta r$, we get

$\frac{\partial u}{\partial t}=D \frac{\partial u^{2}}{\partial r}$

The transformation to the variable $\theta$ leads to zero initial conditions that allows to apply the Riemann-Liouville fractional semi-derivatives after splitting (3a) $\mathrm{as}^{25}$

$\frac{\partial^{1 / 2} u}{\partial t^{1 / 2}}=-\sqrt{D} \frac{\partial u}{\partial r}$

Here

$\frac{\partial^{1 / 2} u}{\partial t^{1 / 2}}=\frac{1}{\Gamma(1 / 2)} \frac{d}{d t} \int_{0}^{t} \frac{u_{r=R}}{\sqrt{u-\tau}} d t$

is a half-time fractional derivative in the Riemann-Liouville sense [25], and $\Gamma(\bullet)$ is the Gamma function, where $\Gamma(1 / 2)=\sqrt{\pi}$.

At $r=R$ we have from (3c):

$-\left.\frac{\partial u}{\partial r}\right|_{r=R}=\left.\frac{1}{\sqrt{D}} D_{t}^{1 / 2} u\right|_{r=R}-\frac{u_{0}}{\sqrt{\pi t}}$

Since, $\left.u\right|_{r=R}=R \theta^{*}=\left.R\left(C-C^{*}\right)\right|_{C=C^{*}}=0$, we get from (4a):

$-\left.\frac{\partial u}{\partial r}\right|_{r=R}=\frac{1}{\sqrt{D} \sqrt{\pi t}} R\left(\theta_{0}-\theta^{*}\right)=\frac{1}{\sqrt{D} \sqrt{\pi t}} R\left(C_{0}-C^{*}\right), \theta^{*}=0$

With $u^{*}=\theta^{*} R, u_{0}=\theta_{0} R$ the boundary flux $(\partial C / \partial r)_{r=R}=N_{r=R}$ is given by

$-(\partial u / \partial r)_{r=R}=\frac{1}{\sqrt{D}} \frac{\partial^{1 / 2} u}{\partial t^{1 / 2}}-\frac{u_{0}}{\sqrt{\pi t}}=\frac{1}{\sqrt{D}} \frac{\left(u^{*}-u_{0}\right)}{\sqrt{\pi t}}$

On the other hand, we have $\theta=C-C^{*}$ and $u=r \theta$, as well as

$\frac{\partial u}{\partial r}=\theta+r \frac{\partial \theta}{\partial r}$

$\left.\frac{\partial u}{\partial r}\right|_{r=R}=\left.R \frac{\partial C}{\partial r}\right|_{r=R}-\left(C_{0}-C^{*}\right)$

Equating the right-hand sides of (5a) and (5b6) we get $-\left.\frac{\partial C}{\partial r}\right|_{r=R}=-\frac{\left(C^{*}-C_{0}\right)}{R}\left(1+\frac{R}{\sqrt{\pi} \sqrt{D t}}\right)$

Now with (6a) and (1b) we read

$N_{r=R}=-\frac{D}{R} \frac{\left(C^{*}-C_{0}\right)}{1-C^{*} / \rho_{0}}\left(1+\frac{R}{\sqrt{\pi} \sqrt{D t}}\right)$

Expressing $(\partial C / \partial r)_{r=R}$ from (4a) and by the transforms $\theta=C-C^{*}$ and $u=r \theta$ we get
$N_{r=R}=\frac{D}{R} S\left[1+\frac{1}{\sqrt{\pi}} \frac{R}{\sqrt{D t}}\right], S=\frac{C^{*}-C_{0}}{1-C^{*} / \rho_{0}}$

The expression (7a) is the same as that developed by Rice and $\mathrm{Do}^{24}$. However, the development of the concentration profile in the bulk phase was avoided thanks to the fractional half-time derivative (3c). Moreover, the transform $\theta=C-C^{*}$ zero initial conditions $u_{0}=0$ permits the Riemann-Liouville fractional derivative to be used to relate the flux and the function value at solid-liquid interface. In the next section we develop two approximate solutions starting from the result $(7 \mathrm{a}, 7 \mathrm{~b})$ and the balance equation (1a) but with different scaling and formation of the defenseless variables.

\section{Particle dissolution (Change of particle radius in time)}

\section{Solution - 1}

Now, with the expression (7a) the mass balance equation (1a) reads as

$R \frac{d R}{d t}=-D S\left[1+\frac{1}{\sqrt{\pi}} \frac{R}{\sqrt{D t}}\right]$

Since in the dissolution process the particle size is time-varying and the process has no characteristic length scale we introduce a similarity variable $\eta=R / \sqrt{D t}$ which is a crucial step differing from the approach used by Rice and $\mathrm{Do}^{24}$. Now, with the new variable we get

$\frac{d \eta^{2}}{d t}+\frac{\eta^{2}}{t}=-\frac{2 S}{t}-\frac{1}{t} \frac{S}{\sqrt{\pi}} \eta$

With the substitution $\eta^{2}=y$ we get

$\frac{d y}{d t}+\frac{y}{t}=-\frac{2 S}{t}-\frac{1}{t} \frac{S}{\sqrt{\pi}} \sqrt{y}$

Re-arranging (9b) with $\sqrt{y}=z$ have

$2 \frac{d(\sqrt{y})}{d t}+\frac{\sqrt{y}}{t}=-\frac{1}{t} \frac{A}{\sqrt{y}}-\frac{B}{t}, A=2 S, B=2 S / \sqrt{\pi}$

or more conveniently as a separable equation

$2 \frac{d z}{d t}=-\frac{1}{t}\left(z-\frac{A}{z}-B\right)$

The solution of (10b) (Performed by Maple) is

$z+\frac{B}{2} \ln \left(-z^{2}+B z+A\right)+\frac{\left(2 A+B^{2}\right)}{\sqrt{4 A+B^{2}}} \arctan h\left(\frac{-2 z+B}{\sqrt{4 A+B^{2}}}\right)=-\ln t$

Expressing the time $t$ explicitly from (11a) we get

$$
t=\frac{1}{\exp \left[z+\frac{B}{2} \ln \left(-z^{2}+B z+A\right)+\frac{\left(2 A+B^{2}\right)}{\sqrt{4 A+B^{2}}} \arctan h\left(\frac{-2 z+B}{\sqrt{4 A+B^{2}}}\right)\right]}
$$

At the point of full particle dissolution when $R=0$ we have from the similarity variable $\eta=R / \sqrt{D t}=z=0$. Hence the time of complete dissolution $t_{f}$ is

$t_{f_{1}}=\frac{1}{\exp \left[\frac{B}{2} \ln (A)+\frac{\left(2 A+B^{2}\right)}{\sqrt{4 A+B^{2}}} \arctan h\left(\frac{B}{\sqrt{4 A+B^{2}}}\right)\right]}$

$A=2 S, B=2 S / \sqrt{\pi}$

Therefore, the time for completing the dissolution depends only on solubility $S$ expressed in two terms $A=2 S, B=2 S / \sqrt{\pi}$. Both of them are of equal order 
of magnitude because the solubility factor is a small value with order of magnitude $10^{-3} 24$. In the repeating terms $2 A+B^{2}$ and $4 A+B^{2}$ in (12a) we may neglect $B^{2}$ because its order of magnitude is $10^{-6}$. However, this approximation can yield some errors, and to avoid them, the expression (12a) can be transformed into

$$
t_{f_{1}}=\frac{1}{\exp \left[\frac{B}{2} \ln (A)+B \frac{\left(2 \frac{A}{B^{2}}\right)}{\sqrt{4 \frac{A}{B^{2}}+1}} \arctan h\left(\frac{1}{\sqrt{4 \frac{A}{B^{2}}+1}}\right]\right]}
$$

Now, we have the order of magnitude $\frac{A}{B^{2}}=\frac{\pi}{2 S} \quad O\left(10^{3}\right)$

Now, we may derive some approximate expressions about $t_{f 1}$ through (13a) namely

$$
t_{f}=\frac{1}{\exp \left[\frac{S}{\sqrt{\pi}} \ln (2 S)+\frac{\left(8 S+\frac{4 S^{2}}{\pi}\right)}{\sqrt{8 S+\frac{4 S^{2}}{\pi}}} \arctan h\left(\frac{\frac{2 S}{\sqrt{\pi}}}{\sqrt{8 S+\frac{4 S^{2}}{\pi}}}\right)\right]}
$$

and assuming $\frac{4 S^{2}}{\pi} \leq 8 S$ we get

$$
t_{f_{1}} \approx \frac{1}{\exp \left[\frac{S}{\sqrt{\pi}} \ln (2 S)+\sqrt{8 S} \arctan h\left(\frac{\sqrt{S}}{\sqrt{2 \pi}}\right)\right]}
$$

$$
t_{f 1} \approx \frac{1}{\exp \left[\frac{S}{\sqrt{\pi}} \ln (2 S)\right]}
$$

\section{Solution - 2}

The Solution -1 was developed by introduction of a similarity variable $\eta=R / \sqrt{D t}$ since we followed the classics in diffusion problems ${ }^{29}$ through a semi-infinite medium. However, since the particle radius change in time is an initial value problem with $R=R_{0}(t=0)$ an alternative initial scaling of eq. (8) by introducing characteristic time and length scales ${ }^{24}$ can be applied, namely $r=R / R_{0}$ and $t_{0}=R_{0}{ }^{2} / D, \tau=t / t_{0} \quad(14 \mathrm{a}, 14 \mathrm{~b}, 14 \mathrm{c})$

This leads to a dimensionless form of eq. (8), namely $R \frac{d R}{d t}=-D S\left[1+\frac{1}{\sqrt{\pi}} \frac{R}{\sqrt{D t}}\right] \Rightarrow\left(\frac{R_{0}^{2}}{t_{0}}\right) r \frac{d r}{d \tau}=-D S\left[1+\frac{1}{\sqrt{\pi}} \frac{R_{0}}{\sqrt{t_{0}}} \frac{r}{\sqrt{D \tau}}\right]$

Using (14a, 14b, 14c) we finally read

$\frac{d r^{2}}{d \tau}=-2 S\left[1+\frac{1}{\sqrt{\pi}} \frac{r}{\sqrt{\tau}}\right]$

Introducing the variable $p=r^{2}$ we get

$\frac{d p}{d \tau}=-2 S\left[1+\frac{1}{\sqrt{\pi}} \sqrt{\frac{p}{\tau}}\right]$

The solution of (17) using Maple gives

$\tau-\frac{1}{2}\left(\frac{\pi \ln (\pi-p(t))}{S}\right)-\frac{\sqrt{\pi}}{S} \sqrt{p(t)}+\frac{\pi \operatorname{arctanh}\left(\sqrt{\frac{p(t)}{\pi}}\right)}{S}+C_{1}=0$
Taking into account that at $t=0$ we have $R=R_{0}$, that is equivalent to $\tau=0$ and $r=1$, as well as $p(t=0)=1$, we have for the constant $C_{1}$

$C_{1}=\frac{1}{S}\left[\frac{\pi \ln (\pi-1)}{2}-\sqrt{\pi}+\pi \operatorname{arctanh}\left(\sqrt{\frac{1}{\pi}}\right)\right]$

Thus, we have

$\tau-\left(\frac{\pi \ln (\pi-p(\tau))}{2 S}\right)-\frac{\sqrt{\pi}}{S} \sqrt{p(\tau)}+\frac{\pi \operatorname{arctanh}\left(\sqrt{\frac{p(\tau)}{\pi}}\right)}{S}+$

$+\frac{1}{S}\left[\frac{\pi \ln (\pi-1)}{2}-\sqrt{\pi}+\pi \operatorname{arctanh}\left(\sqrt{\frac{1}{\pi}}\right)\right]=0$

The final dissolution time $\mathrm{t}_{{ }^{2}}$ at $\mathrm{p}(\tau)=0$ (with $\tau=t / t_{0} \Rightarrow t=\tau\left(R_{0}^{2} / D\right)$ is

$t_{f 2} \approx \frac{t_{0}}{S}\left[\frac{\pi}{2} \ln \frac{(\pi-1)}{\pi}-\sqrt{\pi}+\pi \operatorname{arctanh} \sqrt{\frac{1}{\pi}}\right] \Rightarrow t_{f 2} \approx \frac{0.367}{S} t_{0}$

\section{SOME COMMENTS}

The approximate analytical solutions developed in the previous sections resulted in

$$
t_{f_{1}} \approx \frac{1}{\exp \left[\frac{S}{\sqrt{\pi}} \ln (2 S)+\sqrt{8 S} \operatorname{arctanh}\left(\frac{\sqrt{S}}{\sqrt{2 \pi}}\right)\right]}
$$

or

$$
t_{f 1} \approx \frac{1}{\exp \left[\frac{S}{\sqrt{\pi}} \ln (2 S)\right]}
$$

and

$t_{f 2} \approx \frac{1}{S}\left[\frac{\pi}{2} \ln \frac{(\pi-1)}{\pi}-\sqrt{\pi}+\pi \operatorname{arctanh} \sqrt{\frac{1}{\pi}}\right] \Rightarrow t_{f 2} \approx \frac{0.367}{S} t_{0}$

These solutions are comparable to the solution of Rice and $\mathrm{Do}^{24}$

$\tau_{f}=\frac{1}{2 S} \exp \left\{\left(-\frac{2 S}{\sqrt{2 \pi S-S^{2}}}\right)\left[\frac{\pi}{2}-\arctan \left(\frac{S}{\sqrt{2 \pi S-S^{2}}}\right)\right]\right\}$

For example, the approximate solution of Rice and Do [24] yields $t_{f 2} \approx \frac{0.5}{S} t_{0}$ close to (21).

\section{CONCLUSIONS}

The present work demonstrates how the fractional half-time derivative in the Riemann-Liouville sense can be applied for direct derivation of the equation governing the time-dependent radius of dissolving solid sphere in an unbounded stagnant medium. The final outcomes are approximate relationships for the time-to-complete dissolution, i.e. the lifetime of the dissolving solids.

The two solutions developed are expressed in terms including the solubility factor starting from the formulation of Rice and $\mathrm{Do}^{24}$. The $1^{\text {st }}$ solution is developed by an introduction of a similarity variable $\eta=R / \sqrt{D t}$ while the $2^{\text {nd }}$ solution applies the classical scaling using 
the initial sphere radius as a length scale that leads to the dimensionless radius $r=R / R_{0}$ and time $\tau=D t / R_{0}{ }^{2}$.

\section{LITERATURE CITED}

1. Noyes, A. \& Whitney, W.R. (1987). The Rate of Solution of Solid Substances in their own Solutions, J. Am. Chem. Soc. 19 (12), 930-934.

2. Higuchi,W.I. \& Hiestand, E.N. (1963). Dissolution rates of finely divided powders I. Effect of a distribution of particle sizes in a diffusion-controlled process. J. Pharm. Sci., 52 (1), 67-71. DOI: $10.1002 / j p s .2600520114$

3. Siepmann, J. \& Siepmann, F. (2011). Mathematical modeling of drug release from lipid dosage formsInt. J. Pharmaceutics, 418 (1), 42-53. DOI: 10.1016/j.ijpharm.2011.07.015.

4. Haverkamp, R.G. \& Welch, B.J. (1998). Modelling the dissolution of alumina powder in cryolite, Chem. Eng. Proc. 37 (2), 177-187. http://dx.doi.org/10.1016/S0255-2701(97)00048-2

5. Dorozhkin, S.V. (1996). Fundamentals of the wet-process phosphoric acid production.1. Kinetics and mechanism of the phosphate rock dissolution, Ind. Eng. Chem. Res. 35 (11), 4328-4335. DOI: 10.1021/ie960092u.

6. Bechtloff, N., Justen, P. \& Ulrich, J.(2001). The kinetics of heterogeneous solid-liquid reaction crystallizations-an overview and examples, Chem. Ing. Tech. 73 (5), 453-460. DOI: 10.1002/1522-2640(200105)73:5.

7. Forryan, C.L., Klymenko, O.V., Wilkins, S.J., Brennan, C.M. \& Compton, R.G. (2005). Experimental and Theoretical Study of the Surface-Controlled Dissolution of Cylindrical Particles. Application to Solubilization of Potassium Hydrogen Carbonate in Hot Dimethylformamide, J. Phys. Chem. B, 109 (44), 20786-20793. DOI:10.1021/jp058197a.

8. Lu, T.K., Frisella, M.E. \& Johnson, K.C. (1993). Dissolution modeling: Factors affecting the dissolution rates of polydisperse powders, Pharm Res 10 (9), 1308-1314. DOI: 10.1023/A:1018917729477.

9. Liu, B.T. \& Hsu, J.P. (2006). Theoretical analysis on diffusional release from ellipsoidal drug delivery devices, Chem. Eng. Sci., 61 (6) 1748-1752. http://dx.doi.org/10.1016/j. ces.2005.10.014

10. Sertsou, G. (2004). Analytical Derivation of Time Required for Dissolution of Monodisperse Drug Particles, J. Pharm. Sci., 93 (8), 1941-1943. DOI: 10.1002/jps.20119.

11. Costa, P. \& Lobo, J.M.S. (2001). Review: modeling and comparison of dissolution profiles, Europ. J. Pharmac. Sci. 13 (2), 123-133. DOI: 10.1016/S0928-0987(01)00095-1.

12. Allers, T., Luckas, M. \& Schmidt, K.G. (2003). Modeling and measurement of the dissolution rate of solid particles in aqueous suspensions. Part I. Modeling, Chem. Eng. Technol. 26 (11), 1131-1136. DOI: 10.1002/ceat.200303007.

13. Marabi, A., Mayor, G., Burbidge, A., Wallach, R. \& Saguy, I.S. (2008). Assessing dissolution kinetics of powders by a single particle approach, Chem. Eng. J. 139 (1), 118-127. DOI: 10.1016/j.cej.2007.07.081.

14. Rakoczy, R. \& Masiuk, S. (2011). Studies of a mixing process induced by a transverse rotating magnetic field, Chemical Engineering Science, 66 (11), 2298-2308. DOI: 10.1016/j. ces.2011.02.021.

15. Rakoczy, R. (2013). Mixing energy investigations in a liquid vessel that is mixed by using a rotating magnetic field, Chemical Engineering and Processing: Process Intensification, 66, April, 1-11. DOI: 10.1016/j.bbr.2011.03.031.012.

16. Crank, J. (1975). The Mathematics of Diffusion. 2nd ed., Oxford University Press, London.

17. van Keer, R. \& Kacur, J. (1998). On a numerical model for diffusion-controlled growth and dissolution of spherical precipitates, Mathematical Problems in Engineering, 4 (2), 115-133. ISSN: 1024123X.
18. Vermolen, F.J., van Mourik, P. \& van der Zwaag, S. (1997). Analytical approach to particle dissolution in a finite medium, Mater. Sci. Technol. 13 (4), 308-312. ISSN: 02670836.

19. Vrentas, J.S. \& Shin, D. (1980). Perturbation solutions of spherical moving boundary problems. II, Chem. Eng. Sci. 35 (8), 1697-1705. DOI: 10.1016/0009-2509(80)85004-4.

20. Vrentas, J.S. \& Shin, D. (1980). Perturbation solutions of spherical moving boundary problems. I, Chem. Eng. Sci. 35 (8) 1687-1696. DOI: 10.1016/0009-2509(80)85003-2.

21. Asthana, R. \& Pabi, S.K. (1990). An Approximate Solution for the Finite-extent Moving-boundary Diffusion-controlled Dissolution of Spheres, Materials Science and Engineering, A128 (2), 253-258. DOI: 10.1016/0921-5093(90)90233-S.

22. Kupiec, K. \& Gwadera, M. (2012). On the application of an approximate kinetic equation of heat and mass transfer processes: the effect of body shape, Heat Mass Transfer, 48 (4), 599-610. DOI: 10.1007/s00231-011-0905-6.

23. Rosner, D.E. (1969). Lifetime of a Highly Soluble Dense Spherical Particle1, The Journal of Physical Chemistry, 73 (2), 382-387. DOI: 10.1021/j100722a019.

24. Rice, R.G. \& Do, D.D. (2006). Dissolution of a solid sphere in an unbounded, stagnant liquid, Chem. Eng. Sci., 61 (2), 775-778. DOI: 10.1016/j.bbr.2011.03.031.

25. Oldham, K.B. \& Spanier, J. (1974). The Fractional calculus, New York, USA, Academic Press.

26. dos Santos, M.C., Lenzi, E., Gomes, E.M., Lenzi, M.K. \& Lenzi, E.K. (2011). Development of Heavy Metal sorption Isotherm Using Fractional Calculus, Int. Rev. Chem. Eng., 3 (6), 814-817.

27. Pfaffenzeller, R.A., Lenzi, M.K. \& Lenzi, E.K. (2011). Modeling of Granular Material Mixing Using Fractional Calculus, Int. Rev. Chem. Eng., 3 (6), 818-821.

28. Hristov, J. (2012). Impedance at the Interface of Contacting Bodies: 1-D example solved by semi-derivatives, Thermal Science, 16, 623-627. DOI: 10.2298/TSCI111125017H.

29. Carslaw, H.S. \& Jaeger, J.C. (1959). Conduction of Heat in Solids, London, UK, Oxford University Press. 